\title{
Formulação variacional e estimativas a priori para o método de Galerkin para uma equação de difusão fracionária
}

\author{
Maria E. S. Lima ${ }^{1}$ \\ Imecc/Unicamp, Campinas, SP \\ Edmundo C. Oliveira ${ }^{2}$ \\ Imecc/Unicamp, Campinas, SP \\ Arlúcio C. Viana ${ }^{3}$ \\ Dma/Ufs, São Cristóvão, SE
}

Resumo. Neste trabalho abordamos algumas ferramentas que possibilitam aplicar o metódo de Galerkin para uma equação de difusão fracionária, determinando estimativas a priori e formulação variacional que nos permitem garantir existência e unicidade de uma solução.

Palavras-chave. Método de Galerkin, equação de difusão fracionária, estimativas a priori, existência e unicidade de soluções.

\section{Introdução}

O cálculo fracionário tem ganhado muito destaque nas últimas décadas, devido as suas aplicações em diferentes campos da ciência, em particular, na engenharia, fornecendo várias ferramentas úteis para resolver equações diferenciais e integrais e outros problemas envolvendo funções especiais da física matemática, além de suas extensões e generalizações em uma e mais variáveis. Dentre as várias aplicações do cálculo fracionário podemos citar o fluxo de um fluido, reologia, processos dinâmicos em estruturas auto-semelhantes e porosas, transporte difusivo semelhante à difusão, redes elétricas, probabilidade e estatística, teoria de controle de sistemas dinâmicos e viscoelasticidade (ver [6]).

Vamos discutir o seguinte problema, equação de difusão e condição inicial

$$
\begin{cases}u_{t}+\partial_{t}\left(g_{\alpha} *(-\Delta)^{\gamma} u\right)=f, & \Omega \times[0, T], \\ u(x, 0)=u_{0}(x), & \Omega,\end{cases}
$$

em que $\alpha \in \mathbb{R}, 0<\alpha<1, T>0,0<\gamma<1, \Omega$ é um domínio limitado suave de $\mathbb{R}^{n}, u=0$ na fronteira de $\Omega$, * denota o produto de convolução e $g_{\alpha}$ é a função de Gel'fand Shilov definida por

$$
g_{\alpha}(t)= \begin{cases}\frac{t^{\alpha-1}}{\Gamma(\alpha)}, & t>0, \\ 0, & t \leq 0,\end{cases}
$$

\footnotetext{
1elismara_sousa@hotmail.com

${ }^{2}$ capelas@unicamp.br

3 arlucioviana@gmail.com
} 
onde $\Gamma$ é a função gama de Euler. Além disso, o operador Laplaciano fracionário pode ser definido na sua forma espectral por (ver seção 2.5.1 de [7]):

$$
(-\Delta)^{\gamma} u(x):=\sum_{k=1}^{\infty} \lambda_{k}^{\gamma}\left(u, e_{k}\right)_{L^{2}(\Omega)} e_{k}(x),
$$

onde $\gamma \in(0,1), \lambda_{k}$ são autovalores, e $e_{k}$ são autofunções de $(-\Delta)$ com condições de contorno de Dirichlet, ou seja,

$$
\begin{aligned}
-\Delta e_{k} & =\lambda_{k} e_{k}, \text { em } \Omega, \\
e_{k} & =0, \text { sobre } \partial \Omega .
\end{aligned}
$$

As equações de difusão de ordem fracionária descrevem fenômenos de difusão anômala, que auxiliam na análise de sistemas como: difusão em plasmas, difusão em fractais, difusão anômala em superfícies líquidas, análise de histogramas de batidas do coração em indivíduos saudáveis, entre outros sistemas físicos (ver [1] e [2]).

A difusão anômala pode ser caracterizada tanto por admitir saltos do tipo Levy flights, representados matematicamente pelo Laplaciano fracionário, como também long rests descritos pela derivada fracionária. Neste caso, a equação apropriada, segundo Schneider e Wyss [9] e Metzler e Klafter [8], é dada por

$$
u_{t}+D_{t}^{1-\alpha}(-\Delta)^{\gamma} u=0,
$$

onde $\gamma \in(0,1)$ e $D_{t}^{\beta} \varphi$ denota a derivada fracionária de $\varphi$ de ordem $\beta>0$ no sentido de RiemannLiouville, ou seja, $\alpha \in(0,1)$ (ver definição abaixo). Dessa maneira, (5) pode ser reescrita como a equação

$$
u_{t}+\partial_{t} \int_{0}^{t} g_{\alpha}(t-s)(-\Delta)^{\gamma} u(s) d s=0
$$

onde $g_{\alpha}$ é a função definida em (2).

Para a formulação variacional do problema vamos usar a forma integral de (1), dada por

$$
u=u_{0}-g_{\alpha} *(-\Delta)^{\gamma} u+1 * f .
$$

Vamos abordar a formulação variacional e demonstrar uma estimativa a priori das soluções aproximadas da forma integral de (1), dada por (7), resultados que servirão para aplicar o método de Galerkin (ver [4]), que consiste em encontrar soluções aproximadas para o problema, projetandoo em subespaços de dimensão finita, lidando com equações diferenciais lineares de ordem fracionária com valores iniciais.

\section{Preliminares}

Nessa seção apresentamos algumas definições e notações para o presente trabalho.

Definição 1. Seja $\Omega=[a, b](-\infty<a<b<\infty)$ um intervalo finito sobre os reais $\mathbb{R}$. As integrais fracionárias de Riemann-Liouville, $I_{a^{+}}^{\alpha}$ e $I_{b^{-}}^{\alpha}$ de ordem $\alpha \in \mathbb{R}(\alpha>0)$ são dadas por:

$$
\begin{aligned}
& \left(I_{a^{+}}^{\alpha} f\right)(t)=\frac{1}{\Gamma(\alpha)} \int_{a}^{t}(t-s)^{\alpha-1} f(s) d s, t>a, \\
& \left(I_{b^{-}}^{\alpha} f\right)(t)=\frac{1}{\Gamma(\alpha)} \int_{t}^{b}(s-t)^{\alpha-1} f(s) d s, t<b,
\end{aligned}
$$

onde $\Gamma(\alpha)$ é a função gama e $f \in L^{1}[a, b]$. 
Definição 2. As derivadas fracionárias de Riemann-Liouville, $D_{a^{+}}^{\alpha}$ e $D_{b^{-}}^{\alpha}$ de ordem $\alpha \in \mathbb{R}(\alpha>0)$ são dadas por:

$$
\begin{array}{r}
\left(D_{a^{+}}^{\alpha} f\right)(t)=\left(\frac{d}{d t}\right)^{n}\left(I_{a^{+}}^{n-\alpha} f\right)(t), \quad(n=[\alpha]+1, t>a), \\
\left(D_{b^{-}}^{\alpha} f\right)(t)=(-1)^{n}\left(\frac{d}{d t}\right)^{n}\left(I_{b^{-}}^{n-\alpha} f\right)(t), \quad(n=[\alpha]+1, t<b),
\end{array}
$$

onde $[\alpha]$ significa a parte inteira de $\alpha$ e $f: I \rightarrow \mathbb{R}$. Tomamos $n=\alpha$, se $\alpha \in \mathbb{N}_{0}$.

Definição 3. A derivada fracionária de Caputo de ordem $\alpha$, em um intervalo $[a, b] \subset \mathbb{R}$, é dada por

$$
\left({ }^{c} D_{a^{+}}^{\alpha} \varphi\right)(t):=\left[D_{a^{+}}^{\alpha}\left(\varphi(s)-\sum_{k=0}^{n-1} \frac{\varphi^{(k)}(a)}{k !}(s-a)^{k}\right)\right](t),
$$

onde $n=[\alpha]+1$ se $\alpha \notin \mathbb{N}$ e $n=\alpha$, se $\alpha \in \mathbb{N}_{0}$

Note que o problema (1) pode ser reescrito como

$$
\begin{cases}u_{t}+D_{t}^{1-\alpha}\left(-\Delta_{x}\right)^{\gamma} u=f, & \Omega \times[0, T], \\ u(x, 0)=u_{0}(x), & \Omega .\end{cases}
$$

Onde $0<\gamma<1$ e $0<\alpha<1$. De fato, uma vez que $0<\alpha<1$ e $1-\alpha<1$, temos que

$$
\begin{aligned}
D_{t}^{1-\alpha}\left[(-\Delta)^{\gamma} u\right] & =\frac{d}{d t}\left(I^{1-(1-\alpha)}(-\Delta)^{\gamma} u\right)=\frac{d}{d t}\left(\frac{1}{\Gamma(\alpha)} \int_{0}^{t}(t-s)^{\alpha-1}(-\Delta)^{\gamma} u(s) d s\right) \\
& =\partial_{t}\left(\int_{0}^{t} g_{\alpha}(t-s)(-\Delta)^{\gamma} u(s) d s\right)=\partial_{t}\left(g_{\alpha} *(-\Delta)^{\gamma} u\right) .
\end{aligned}
$$

Vamos utilizar os seguintes espaços $L^{\infty}\left(0, T ; L^{2}(\Omega)\right), L^{2}\left(0, T ; H^{\gamma}(\Omega)\right)$ e $L^{1}\left(0, T ; L^{2}(\Omega)\right)$, onde $\Omega$ é um aberto do $\mathbb{R}^{n}$, que pode ser representado de modo geral por $L^{p}(0, T ; X)$ em que $X$ é um espaço de Banach com a norma $\|\cdot\|_{X}$. Lembrando que $L^{p}(\Omega)$ é o espaço de todas as funções mensuráveis $f: \Omega \rightarrow \mathbb{R}$, com $\|f\|_{L^{p}(\Omega)}<\infty$ tal que

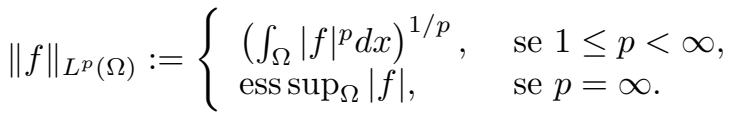

O espaço de Sobolev fracionário $H^{\gamma}$ é um espaço de Hilbert e, é definido a seguir (ver definição A.5 de [7]).

Definição 4. Para qualquer $\gamma \geq 0$

$$
H^{\gamma}(\Omega):=\left\{u=\sum_{k=1}^{\infty} u_{k} \phi_{k} \in L^{2}(\Omega):\|u\|_{H^{\gamma}(\Omega)}^{2}:=\sum_{k=1}^{\infty} \lambda_{k}^{\gamma} u_{k}^{2}<\infty\right\},
$$

onde $\left(\lambda_{k}, \phi_{k}\right)$ são os autovalores e seus respectivos autovetores de $(-\Delta)$ com condições de contorno de Dirichlet, cuja a norma coincide com $\left\|(-\Delta)^{\gamma} u\right\|_{L^{2}}$, de acordo com (3).

Definição 5. O espaço $L^{p}(0, T ; X)$ consiste de todas as funções mensuráveis

$$
u:(0, T) \rightarrow X
$$

com

$$
\|u\|_{L^{p}(0, T ; X)}=\left(\int_{0}^{T}\|u(t)\|_{X}^{p} d t\right)^{1 / p}<\infty
$$


para $1 \leq p<\infty, e$

$$
\|u\|_{L^{\infty}(0, T ; X)}=\sup _{t \in(0, T)}\|u(t)\|_{X}<\infty
$$

Por simplicidade, em alguns momentos, denotamos $L^{p}\left(0, T ; L^{p}(\Omega)\right)$ por $L^{p}\left(0, T ; L^{p}\right)$. Além disso, denotamos o produto interno em $L^{2}$ por $(\cdot, \cdot)$ e em $H^{\gamma}$ por $(\cdot, \cdot)_{H^{\gamma}}$.

Utilizamos o seguinte teorema de existência e unicidade para um problema de Cauchy de uma equação matricial fracionária com derivada de Caputo. (ver Teorema 7.14, [6])

Antes, apresentamos algumas definições necessárias.

Definição 6. Sejam $A \in M_{n}(\mathbb{R}), z \in \mathbb{C}$ e $\alpha>0$. Definimos a matriz $\alpha$-função exponencial por

$$
e_{\alpha}^{A z}:=z^{\alpha-1} \sum_{k=0}^{\infty} A^{k} \frac{z^{\alpha k}}{\Gamma[(k+1) \alpha]} .
$$

Definição 7. Um espaço ponderado de funções contínuas é da forma

$$
C_{n-\alpha}[a, b]=\left\{g(x):(x-a)^{n-\alpha} g(x) \in C[a, b],\|g\|_{C n-\alpha}=\left\|(x-a)^{n-\alpha} g(x)\right\|_{C}\right\} \text {. }
$$

Teorema 1. O seguinte problema de valor inicial

$$
\begin{array}{r}
\left({ }^{c} D_{a^{+}}^{\alpha} \bar{Y}\right)(x)=A \bar{Y}(x)+\bar{B}(x), \\
\bar{Y}(a)=\bar{b},\left(\bar{b} \in \mathbb{R}^{n}\right),
\end{array}
$$

onde $A \in M_{n}(\mathbb{R})$ e $\bar{B} \in \bar{C}_{1-\alpha}([a, b])$, tem uma única solução contínua dada por

$$
\bar{Y}=\int_{a}^{x} e_{\alpha}^{A}(x-\xi)[\bar{B}(\xi)+A \bar{b}] d \xi+\bar{b}
$$

Precisamos do seguinte resultado que pode ser encontrado em [3].

Teorema 2. Seja $(H,(\cdot, \cdot))$ um espaço de Hilbert real, $f \in L^{2}(0, T ; H)$ e $\alpha \in(0,1)$. Então

$$
\int_{0}^{T}\left(f(t), g_{\alpha} * f(t)\right) d t \geq 0 .
$$

\section{Resultados principais}

Nesta seção abordamos a formulação variacional e obtemos uma estimativa a priori necessária para aplicar o método de Galerkin de (1).

\subsection{Formulação variacional}

Para a formulação variacional do problema vamos usar a forma integral de (1), dada por

$$
u=u_{0}-g_{\alpha} *(-\Delta)^{\gamma} u+1 * f
$$

onde $u=0$ na fronteira de $\Omega$ e $\gamma \in(0,1)$. Multiplicando $(7)$ por $v \in H^{\gamma}$ tal que $v=v(x)$ e, integrando sobre $\Omega$, temos que

$$
\int_{\Omega} u v d x=\int_{\Omega} u_{0} v d x-\int_{\Omega}\left(g_{\alpha} *(-\Delta)^{\gamma} u\right) v d x+\int_{\Omega}(1 * f) v d x
$$


Assim, utilizando o teorema de Fubini e sabendo que o Laplaciano fracionário é auto-adjunto em $L^{2}$, além de possuir a propriedade de semigrupo, temos

$$
\begin{aligned}
\int_{\Omega}\left(g_{\alpha} *(-\Delta)^{\gamma} u\right) v d x & =\int_{\Omega} \int_{0}^{t} g_{\alpha}(t-s)(-\Delta)^{\gamma} u(s, x) v(x) d s d x \\
& =\int_{0}^{t} g_{\alpha}(t-s) \int_{\Omega}(-\Delta)^{\gamma} u(s, x) v(x) d x d s \\
& =\int_{0}^{t} g_{\alpha}(t-s) \int_{\Omega}(-\Delta)^{\frac{\gamma}{2}} u(s, x)(-\Delta)^{\frac{\gamma}{2}} v(x) d x d s \\
& =\int_{\Omega}\left(\int_{0}^{t} g_{\alpha}(t-s)(-\Delta)^{\frac{\gamma}{2}} u(s, x) d s\right)(-\Delta)^{\frac{\gamma}{2}} v(x) d x \\
& =\int_{\Omega}\left(g_{\alpha} *(-\Delta)^{\frac{\gamma}{2}} u\right)(-\Delta)^{\frac{\gamma}{2}} v d x \\
& =\left(g_{\alpha} *(-\Delta)^{\frac{\gamma}{2}} u,(-\Delta)^{\frac{\gamma}{2}} v\right) .
\end{aligned}
$$

Desse modo, segue de (17) que

$$
(u, v)=\left(u_{0}, v\right)-\left(g_{\alpha} *(-\Delta)^{\frac{\gamma}{2}} u,(-\Delta)^{\frac{\gamma}{2}} v\right)+(1 * f, v)
$$

ou

$$
(u, v)=\left(u_{0}, v\right)-\left(g_{\alpha} * u, v\right)_{H^{\gamma}}+(1 * f, v),
$$

onde (19) nos dá a forma variacional do problema. Denotamos $\left(g_{\alpha} * u, v\right)_{H^{\gamma}}$ por $B[u, v ; t]$. Agora vamos construir soluções aproximadas. Para isto, considere uma base $\left\{v_{k}\right\}_{k}$ ortogonal de $H^{\gamma} \mathrm{e}$ ortonormal de $L^{2}(\Omega)$.

Para cada $m$ inteiro, considere o subespaço vetorial

$$
V^{m}=\left[v_{1}, \cdots, v_{m}\right]
$$

e,

$$
u_{m}(t)=\sum_{j=1}^{m} \beta_{m}^{j}(t) v_{j},
$$

onde devemos determinar os coeficientes $\beta_{m}^{j}(t)(0 \leq t \leq T$ e $j=1, \cdots, m)$ tais que

$$
\beta_{m}^{j}(0)=\left(u_{0}, v_{j}\right) \quad j=1, \cdots, m
$$

$$
\left(u_{m}, v_{j}\right)=\beta_{m}^{j}(0)-B\left[u_{m}, v_{j} ; t\right]+\left(1 * f, v_{j}\right) .
$$

Teorema 3. Se $f \in L^{\infty}\left(0, T ; L^{2}\right)$, então para cada inteiro $m=1,2, \cdots$, existe uma única função $u_{m}$ da forma (20) satisfazendo (21) e (22).

Demonstração. Suponha que $u_{m}$ tenha a forma (20). A demonstração se reduz a mostrar a existência e unicidade dos $\beta_{m}^{j}(t)$. Daí,

$$
\left(u_{m}, v_{k}\right)=\left(\sum_{j=1}^{m} \beta_{m}^{j}(t) v_{j}, v_{k}\right)=\beta_{m}^{k}(t),
$$

pois $\left\{v_{j}\right\}_{j}$ é ortonomal. Além disso, 


$$
\begin{aligned}
B\left[u_{m}, v_{k} ; t\right] & =\left(g_{\alpha} *(-\Delta)^{\frac{\gamma}{2}} u_{m},(-\Delta)^{\frac{\gamma}{2}} v_{k}\right)=g_{\alpha} *\left((-\Delta)^{\frac{\gamma}{2}} \sum_{j=1}^{m} \beta_{m}^{j}(t) v_{j},(-\Delta)^{\frac{\gamma}{2}} v_{k}\right) \\
& =g_{\alpha} *\left(\sum_{j=1}^{m} \beta_{m}^{j}(t)(-\Delta)^{\frac{\gamma}{2}} v_{j},(-\Delta)^{\frac{\gamma}{2}} v_{k}\right)=\sum_{j=1}^{m}\left(g_{\alpha} * \beta_{m}^{j}\right)(t)\left(v_{j}, v_{k}\right)_{H^{\gamma}} \\
& =\sum_{j=1}^{m}\left(g_{\alpha} * \beta_{m}^{j}\right)(t) e^{j k} .
\end{aligned}
$$

Defina $f^{k}(t)=\left(1 * f(t), v_{k}\right)$. Logo, de $(22)$, temos

$$
\beta_{m}^{k}(t)-\beta_{m}^{j}(0)+\sum_{j=1}^{m} e^{j k}\left(g_{\alpha} * \beta_{m}^{j}\right)(t)=f^{k}(t)
$$

onde $e^{j k}=\left(v_{j}, v_{k}\right)$.

$$
\text { Sejam } X=\left[\begin{array}{c}
\beta_{m}^{1}(t) \\
\vdots \\
\beta_{m}^{n}(t)
\end{array}\right], X^{0}=\left[\begin{array}{c}
\beta_{m}^{1}(0) \\
\vdots \\
\beta_{m}^{n}(0)
\end{array}\right], A=\left[e^{i j}\right] \text { e } F=\left[\begin{array}{c}
\left(f, v_{1}\right) \\
\vdots \\
\left(f, v_{m}\right)
\end{array}\right] \text { podemos reescrever }
$$

(23) na seguinte forma matricial

$$
X-X^{0}+g_{\alpha} *(A X)=1 * F,
$$

convoluindo com $g_{1-\alpha}$, segue que

$$
g_{1-\alpha} *\left(X-X^{0}\right)+1 *(A X)=1 * g_{1-\alpha} * F \Rightarrow^{c} D^{\alpha} X+A X=g_{1-\alpha} * F .
$$

Assim, por hipótese, como $f \in L^{\infty}\left(0, T ; L^{2}\right)$, segue que $g_{1-\alpha} * F \in C_{1-\alpha}([0, T])$. Logo, pelo teorema 1 segue a existência e unicidade dos $\beta_{m}^{j}$.

\subsection{Estimativas a priori}

Nesta seção demonstramos uma estimativa a priori dada pelo teorema a seguir.

Teorema 4. Seja $\alpha \in(0,1)$. Se $f \in L^{1}\left(0, T ; L^{2}\right)$, então

$$
\left\|u_{m}\right\|_{L^{\infty}\left(0, T ; L^{2}\right)} \leq\left\|u_{0 m}\right\|_{L^{2}}+\|f\|_{L^{1}\left(0, T ; L^{2}\right)} .
$$

Se, adicionalmente, $f \in L^{\infty}\left(0, T ; L^{2}\right)$, então

$$
\left\|u_{m}\right\|_{L^{1}\left(0, T ; H^{\gamma}\right)} \leqslant \frac{T^{2-\alpha}}{\Gamma(2-\alpha)}\left\|u_{0 m}\right\|_{L^{2}}+\frac{T^{3-\frac{\alpha}{2}}}{\Gamma(2-\alpha)^{\frac{3}{2}} \Gamma(\alpha+1)}\|f\|_{L^{\infty}\left(0, T ; L^{2}\right)} .
$$

Demonstração. Sendo $u_{m}$ a função definida em (20) e garantida pelo teorema 3, multiplicamos (18) por $\beta_{m}^{j}$ e somamos com $j$ variando de 1 a $m$, para obter

$$
\left\|u_{m}\right\|_{L^{2}}^{2}=\left(u_{0 m}, u_{m}\right)-\left(g_{\alpha} * u_{m}, u_{m}\right)_{H^{\gamma}}+\left(1 * f, u_{m}\right)_{L^{2}} .
$$

Notamos que $u_{m} \in L^{2}\left(0, T ; H^{\gamma}\right)$. De fato, olhando para a expressão (20) podemos inferir que

$$
\left\|u_{m}(t)\right\|_{L^{2}\left(0, T ; H^{\gamma}\right)}^{2} \leq \sum_{j=1}^{m} \int_{0}^{T}\left\|\beta_{m}^{j}(t) v_{j}(\cdot)\right\|_{H^{\gamma}}^{2} d t \leq \sum_{j=1}^{m} \int_{0}^{T}\left|\beta_{m}^{j}(t)\right|^{2} d t\left\|v_{j}\right\|_{H^{\gamma}}
$$




$$
=\sum_{j=1}^{m}\left\|\beta_{m}^{j}(t)\right\|_{L^{2}(0, T)}^{2}\left\|v_{j}\right\|_{H^{\gamma}}<\infty,
$$

uma vez que $\beta_{m}^{j} \in L^{2}(0, T), v_{j} \in H^{\gamma}$ e a soma é finita.

Assim, pelo teorema 2, temos

$$
\left(g_{\alpha} * u, u\right)_{H^{\gamma}} \geq 0
$$

Segue-se disso e de (26) que

$$
\left\|u_{m}\right\|_{L^{2}}^{2} \leq\left(u_{0 m}, u_{m}\right)_{L^{2}}+\left(1 * f, u_{m}\right)_{L^{2}} \leq\left\|u_{0 m}\right\|_{L^{2}}\left\|u_{m}\right\|_{L^{2}}+\|f\|_{L^{1}\left(0, T ; L^{2}\right)}\left\|u_{m}\right\|_{L^{2}},
$$

pela desigualdade de Hölder. Daí,

$$
\left\|u_{m}(t)\right\|_{L^{2}} \leq\left\|u_{0 m}\right\|_{L^{2}}+\|f\|_{L^{1}\left(0, T ; L^{2}\right)} .
$$

Isto prova (24). Para a demonstração de (25) é utilizado o lema 6.2 (ver [5]) e a desigualdade integral de Minkowski.

\section{Conclusões}

Neste trabalho obtivemos a formulação variacional e uma estimativa a priori de (9), resultados que vão nos auxiliar para aplicar o método de Galerkin e nos possibilitar a existência e unicidade da solução do problema (9). Estudos nesse sentido encontram-se em andamento.

\section{Referências}

[1] Biler,P., Funaki T. and Woyezynski, W. A. Fractal Burgers equations, 1: 9-46, 1998. MR1637513

[2] Bonkile, M., Awasthi,A., Lashmi, C., Mukundai,V. and Aswin,V. S. A systematic literature review of Burgers' equation with recent advances, Pranama J. Phys. 90:60, 2018.

[3] Djilali, L.,Rougirel, A.Galerkin method for time fractional difusion equations Journal of Elliptic and Parabolic Equations 4:349-368, 2018.

[4] Evans, L. C. Partial differential equations. Providence, RI, 1998

[5] Kemppainen, J., et al. Decay estimates for time-fractional and other non-local in time subdiffusion equations in $\mathbb{R}^{d}$. Mathematische Annalen 366: 941-979, 2016.

[6] Kilbas, A. A., Srivastava, H. M. and Trujillo, J. J. Theory and Applications of Fractional Differential Equations. Elsevier Science B.V., Amsterdam, 2006.

[7] Lischke, A. et al. What is the fractional Laplacian? A comparative review with new results. Journal of Computational Physics, 404:1-62, 2020.

[8] Metzler R. and Klafter, J. The random walks guide to anomalous diffusion: A fractional dynamics approach, Phys. Rep., 339:1-77, 2000.

[9] Schneider, W. R. and Wyss, W. Fractional diffusion and wave equations, J. Math. Phys. 30:134-144, 1989. 